\title{
Expanding the Tanh-Function Method for Solving Nonlinear Equations
}

\author{
Nassar Hassan Abdel-All, Mohamed Abd-Allah Abdel-Razek, Abd-Allah Kamel Seddeek \\ Department of Mathematics, Faculty of Science, Assiut University, Assiut, Egypt \\ E-mail: abdel_razek555@yahoo.com \\ Received March 14, 2011; revised June 9, 2011; accepted June 16, 2011
}

\begin{abstract}
In this paper, using the tanh-function method, we introduce a new approach to solitary wave solutions for solving nonlinear PDEs. The proposed method is based on adding integration constants to the resulting nonlinear ODEs from the nonlinear PDEs using the wave transformation. Also, we use a transformation related to those integration constants. Some examples are considered to find their exact solutions such as KdVBurgers class and Fisher, Boussinesq and Klein-Gordon equations. Moreover, we discuss the geometric interpretations of the resulting exact solutions.
\end{abstract}

Keywords: Tanh-Function Method, Nonlinear Equations, Solitary Waves

\section{Introduction}

The importance of nonlinear partial differential equations (PDEs) appears in describing the nonlinear phenomena in various fields of sciences. Many powerful methods have been developed to find the exact solutions of nonlinear PDEs, among them inverse scattering method [1], Hirota bilinear form [2], Painlevé analysis [3], direct algebraic method [4], tanh-function method [5,6] and it's extensions [7-9] and the sine-cosine method [10,11].

Herman et al. [12] introduced a general physical approach to solitary wave construction from linear solutions and obtained many exact solution of nonlinear PDEs using the direct algebraic method [4].

In this paper, we introduce a similar technique to [12] using the tanh-function method to obtain exact solutions for nonlinear evolution and wave equations. The first step in the tanh-function method is using an independent variable to turn the nonlinear PDEs into other nonlinear ordinary differential equations (ODEs) which may or may not be integrable and neglecting the integration constants in case of integrable ODEs. Here, we add integration constants in the resulting integrable nonlinear ODEs. Also, we use a new transformation in which we express the solution function as a sum of another independent function and a constant which are determined later. By means of this modification, we get the exact solutions in which a free constant appears which for some values gives the solutions of the tanh-function method. When the resulting nonlinear ODEs is non integrable, we use the transformation only to get the same exact solutions of the tanh-function method.

\section{Expanding the Tanh-Function Method for Solving Nonlinear Equations}

The tanh-function method, pioneered by Malfliet [5,6], is a common powerful method for solving nonlinear equations. Here, we introduce a modification of the tanhfunction method through the following:

Consider the nonlinear evolution and wave equations in the forms

$$
P\left(u, u_{t}, u_{x}, u_{x x}, \cdots\right)=0, \quad P\left(u, u_{t t}, u_{x}, u_{x x}, \cdots\right)=0,
$$

respectively. Introducing the wave transformation

$$
u(x, t)=U(\xi), \xi=k(x-\omega t),
$$

to change (1) into a nonlinear ODE

$$
O\left(U, U^{\prime}, U^{\prime \prime}, U^{\prime \prime \prime}, \cdots\right)=0,
$$

where $k>0$ is the wave number and $\omega$ is the travelling wave velocity.

Assuming (3) is integrated with respect to $\xi$ as many times as possible without neglecting the integration constants. For the evolution equations the maximum number of integration is 1 and for the wave equations is 2. For reasons that will be explained below, we only leave the integration constant of the last integration. 
To obtain the exact solitary wave solution, possibly having a determined constant term $c_{1}$, we introduce the transformation

$$
U=\phi+c_{1} .
$$

Substituting (4) into (3) and setting the constant part equals to zero in the resulting nonlinear ODE in $\phi$ assuming that the function $\phi$ and its derivatives have the following asymptotic values,

$$
\phi(\xi) \rightarrow \phi_{ \pm} \text {as } \xi \rightarrow \pm \infty,
$$

and for $n \geq 1$

$$
\phi^{(n)}(\xi) \rightarrow 0 \text { as } \xi \rightarrow \pm \infty,
$$

where the superscripts denotes differentiation to the order $n$, with respect to $\xi$, also we assume that $\phi_{ \pm}$ satisfies the algebraic equation in $\phi$, then we get the values of $c_{1}$.

Applying the tanh-function method by introducing the new independent variable $Y=\tanh \xi$ which leads to the change of derivatives in the forms

$$
\begin{aligned}
& \frac{\mathrm{d}}{\mathrm{d} \xi}=\left(1-Y^{2}\right) \frac{\mathrm{d}}{\mathrm{d} Y}, \\
& \frac{\mathrm{d}^{2}}{\mathrm{~d} \xi^{2}}=\left(1-Y^{2}\right)\left(-2 Y \frac{\mathrm{d}}{\mathrm{d} Y}+\left(1-Y^{2}\right) \frac{\mathrm{d}^{2}}{\mathrm{~d} Y^{2}}\right), \cdots
\end{aligned}
$$

and using the finite expansion

$$
\phi(\xi)=S(Y)=\sum_{n=0}^{m} a_{n} Y^{n},
$$

where $m$ is a positive integer determined by the balancing procedure in the resulting nonlinear ODE in $S$. Thus, we have an algebraic system of equations from which the constants $k, \omega, a_{n}(n=0, \cdots, m)$ are obtained and determine the function $\phi$, hence we get the exact solutions of (1).

Now, we obtain exact solutions for some examples of nonlinear evolution and wave equations using the suggested method.

\section{KdV-Burgers Class}

Consider the KdV-Burgers class in the form

$$
u_{t}+\alpha u u_{x}+\beta u_{x x}+\gamma u_{x x x}=0,
$$

where $\alpha, \beta$ and $\gamma$ are real constants. The class (9) gives the Burgers equation and the $\mathrm{KdV}$ equation at $\gamma=0$ and $\beta=0$ respectively.

\subsection{Burgers Equation}

Consider the Burgers equation in the form

$$
u_{t}+\alpha u u_{x}+\beta u_{x x}=0 .
$$

Using (2), for $k=1$, to change (10) into the following nonlinear ODE

$$
-\omega U^{\prime}+\alpha U U^{\prime}+\beta U^{\prime \prime}=0 .
$$

Integrating (11) once to get a new nonlinear ODE in the form

$$
-\omega U+\frac{1}{2} \alpha U^{2}+\beta U^{\prime}+c_{1} C=0,
$$

where $c_{1} C$ is the integration constant.

Introducing (4) into (12), we have

$$
\left(\alpha c_{1}-\omega\right) \phi+\frac{1}{2} \alpha \phi^{2}+\beta \phi^{\prime}+c_{1}\left(\frac{1}{2} \alpha c_{1}+C-\omega\right)=0 .
$$

Using the conditions (5), (6) and that $\phi_{ \pm}$satisfies the algebraic equation

$$
\left(\alpha c_{1}-\omega\right) \phi_{ \pm}+\frac{1}{2} \alpha \phi_{ \pm}^{2}=0,
$$

then the constant term in (13) equals to zero,

$$
c_{1}\left(\frac{1}{2} \alpha c_{1}+C-\omega\right)=0 .
$$

Then we have the following two cases according to the values of $c_{1}$.

Case (1). $c_{1}=0$ :

Using (8), in this case we have

$$
-\omega S+\frac{1}{2} \alpha S^{2}+\beta\left(1-Y^{2}\right) \frac{\mathrm{d} S}{\mathrm{~d} Y}=0 .
$$

Applying the tanh-function method by balancing the nonlinear term $S^{2}$ with the derivative term $\frac{\mathrm{d} S}{\mathrm{~d} Y}$, we get $m=1$, and using (8) we have

$$
\phi(\xi)=S(Y)=a_{0}+a_{1} Y .
$$

Substituting (17) into (16), we obtain

$$
-\omega\left(a_{0}+a_{1} Y\right)+\frac{1}{2} \alpha\left(a_{0}+a_{1} Y\right)^{2}+\beta a_{1}\left(1-Y^{2}\right)=0 .
$$

Setting zero all the coefficients of $Y^{n}(n=0,1,2)$, we get the algebraic system of equations

$$
\begin{aligned}
& -\omega a_{0}+\frac{1}{2} \alpha a_{0}^{2}+\beta a_{1}=0, \\
& -\omega a_{1}+\alpha a_{0} a_{1}=0, \\
& \frac{1}{2} \alpha a_{1}^{2}-\beta a_{1}=0 .
\end{aligned}
$$

From which we have

$$
a_{0}= \pm \frac{2 \beta}{\alpha}, \quad a_{1}=\frac{2 \beta}{\alpha}, \quad \omega= \pm 2 \beta .
$$

Using (4), we get the exact solutions of the tanh- 
function method in the form [13]

$$
u_{1,2}= \pm \frac{2 \beta}{\alpha}+\frac{2 \beta}{\alpha} \tanh (x \mp 2 \beta t),
$$

these solutions represent 2-dimensional surfaces in the Monge form as shown in Figure 1 for $\alpha=\beta=1$.

Case (2). $\quad c_{1}=\frac{2}{\alpha}(\omega-C)$ :

Using the same way as in Case (1), we obtain the exact solutions in the form

$$
u_{1,2}=\frac{2 C \mp 2 \beta}{\alpha}+\frac{2 \beta}{\alpha} \tanh (x-(2 C \mp 2 \beta) t) .
$$

These relations represent surfaces whose Gaussian curvature $K$ and mean curvature $H$ are given by (23)

Thus the solutions (22) represent a family of parabolic surfaces $(K=0, H \neq 0)$, and a family of planes $(K=H=0)$ at $x=(2 C \mp 2 \beta) t$ as shown in Figure 2 for $C=0, \pm 1, \alpha=\beta=1$; when $C=0$, we get the solutions (21).

\subsection{KdV Equation}

Consider the KdV equation in the form

$$
u_{t}+\alpha u u_{x}+\gamma u_{x x x}=0 .
$$

Using (2), for $k=1$, to change (24) into a nonlinear ODE, then integrating once, we obtain

$$
-\omega U+\frac{1}{2} \alpha U^{2}+\gamma U^{\prime \prime}+c_{1} C=0,
$$

where $c_{1} C$ is the integration constant.

Introducing (4) into (25), we get

$$
\left(\alpha c_{1}-\omega\right) \phi+\frac{1}{2} \alpha \phi^{2}+\gamma \phi^{\prime \prime}+c_{1}\left(\frac{1}{2} \alpha c_{1}+C-\omega\right)=0 .
$$

Using the conditions (5), (6) and (14), we get (15), hence we obtain the following two cases according to the values of $c_{1}$.

Case (1). $c_{1}=0$ :

In this case, we get the exact solutions of the tanhfunction method in the forms [14]

$$
\begin{aligned}
& u_{1}=\frac{4 \gamma}{\alpha}-\frac{12 \gamma}{\alpha} \tanh ^{2}(x+4 \gamma t), \\
& u_{2}=\frac{12 \gamma}{\alpha}-\frac{12 \gamma}{\alpha} \tanh ^{2}(x-4 \gamma t),
\end{aligned}
$$

these solutions represent 2-dimensional surfaces in the Monge form as shown in Figure 3 for $\alpha=\gamma=1$.

Case (2). $\quad c_{1}=\frac{2}{\alpha}(\omega-C)$ :

In this Case, we have the exact solutions in the forms

$$
\begin{aligned}
& u_{1}=\frac{2 C+4 \gamma}{\alpha}-\frac{12 \gamma}{\alpha} \tanh ^{2}(x-(2 C-4 \gamma) t), \\
& u_{2}=\frac{2 C+12 \gamma}{\alpha}-\frac{12 \gamma}{\alpha} \tanh ^{2}(x-(2 C+4 \gamma) t) .
\end{aligned}
$$

These relations represent surfaces whose Gaussian curvature $K$ and mean curvature $H$ are given by (29)

Thus the solutions (28) represent a family of parabolic surfaces $(K=0, H \neq 0)$, and a family of planes $(K=H=0)$ at $x=(2 C \mp 4) t+\cosh ^{-1}(2)$ as shown in Figure 4 for $C=0,1, \alpha=\gamma=1$; when $C=0$, we get the solutions (27).

\subsection{KdV-Burgers Equation}

Consider the KdV-Burgers equation in the form:

$$
u_{t}+\alpha u u_{x}+\beta u_{x x}+\gamma u_{x x x}=0 .
$$

Using (2) to change (30) into a nonlinear ODE, then integrating once

$$
-\omega U+\frac{1}{2} \alpha U^{2}+\beta k U^{\prime}+\gamma k^{2} U^{\prime \prime}+c_{1} C=0,
$$

$$
\begin{aligned}
& K_{1,2}=0, \\
& H_{1,2}=\frac{-2 \alpha^{2} \beta\left(1+4 C^{2}-8 C \beta+4 \beta^{2}\right) \operatorname{sech}^{2}(x-(2 C \mp 2 \beta) t) \tanh (x-(2 C \mp 2 \beta) t)}{\left(\alpha^{2}+4 \beta^{2}\left(1+4 C^{2}-8 C \beta+4 \beta^{2}\right) \operatorname{sech}^{4}(x-(2 C \mp 2 \beta) t)\right)^{\frac{3}{2}}}, \\
& K_{1,2}=H_{1,2}=0 \text { at } x=(2 C \mp 2 \beta) t . \\
& K_{1,2}=0, \\
& H_{1,2}=\frac{12 \alpha^{2} \beta\left(1+4 C^{2} \mp 16 C \gamma+16 \gamma^{2}\right) \operatorname{sech}^{4}(x-(2 C \mp 4 \gamma) t)(-2+\cosh (x-(2 C \mp 4 \gamma) t))}{\left(\alpha^{2}+576 \beta^{2}\left(1+4 C^{2} \mp 16 C \gamma+16 \gamma^{2}\right) \operatorname{sech}^{4}(x-(2 C \mp 4 \gamma) t) \tanh (x-(2 C \mp 4 \gamma) t)\right)^{\frac{3}{2}}} \\
& K_{1,2}=H_{1,2}=0 \text { at } x=(2 C \mp 4) t+\cosh ^{-1}(2) .
\end{aligned}
$$



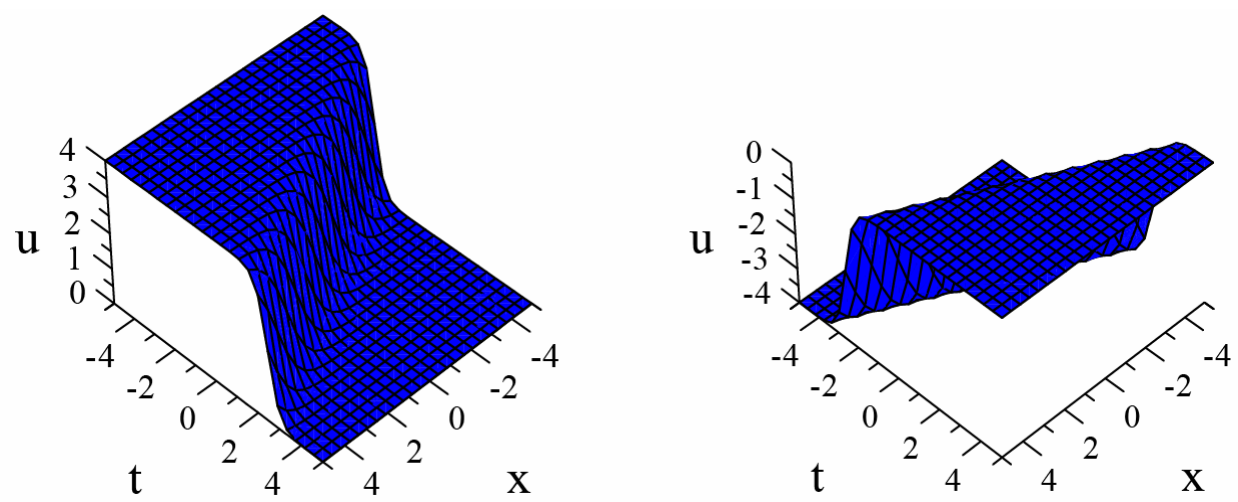

Figure 1. $u_{1}$ and $u_{2}$ in (21).
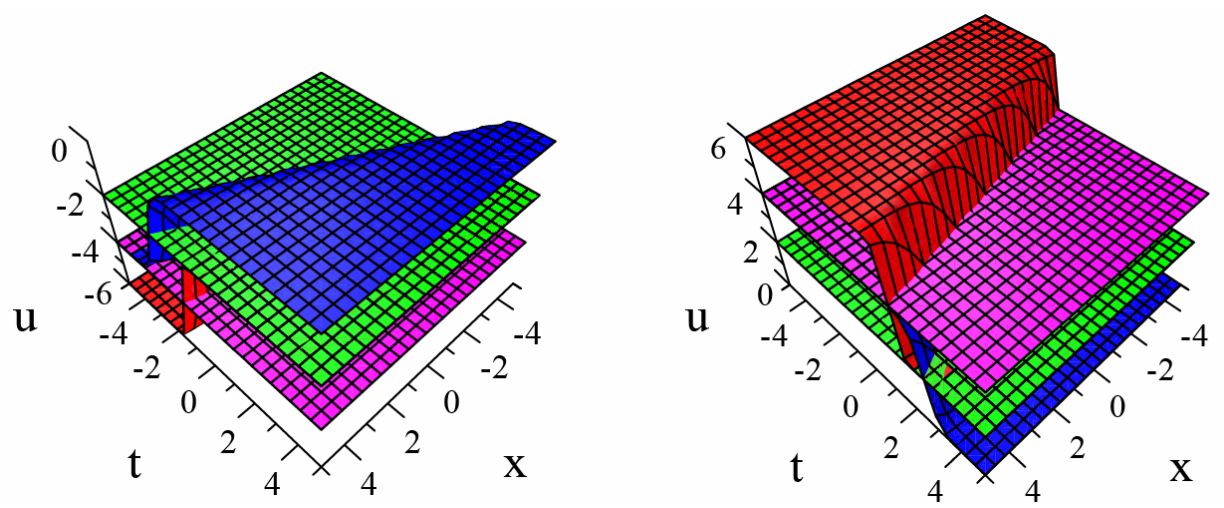

Figure 2. $u_{1}$ and $u_{2}$ in (22).
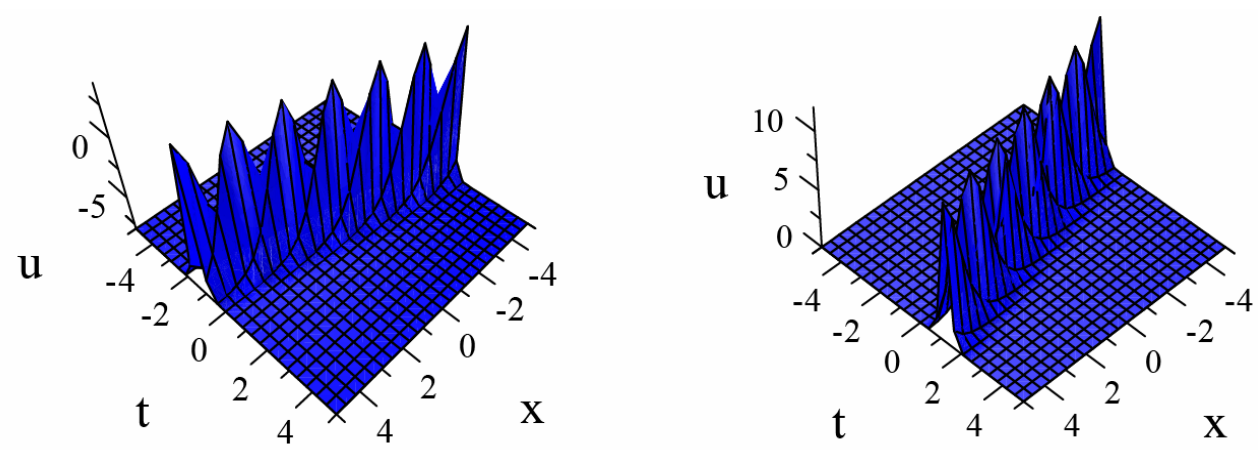

Figure 3. $u_{1}$ and $u_{2}$ in (27).
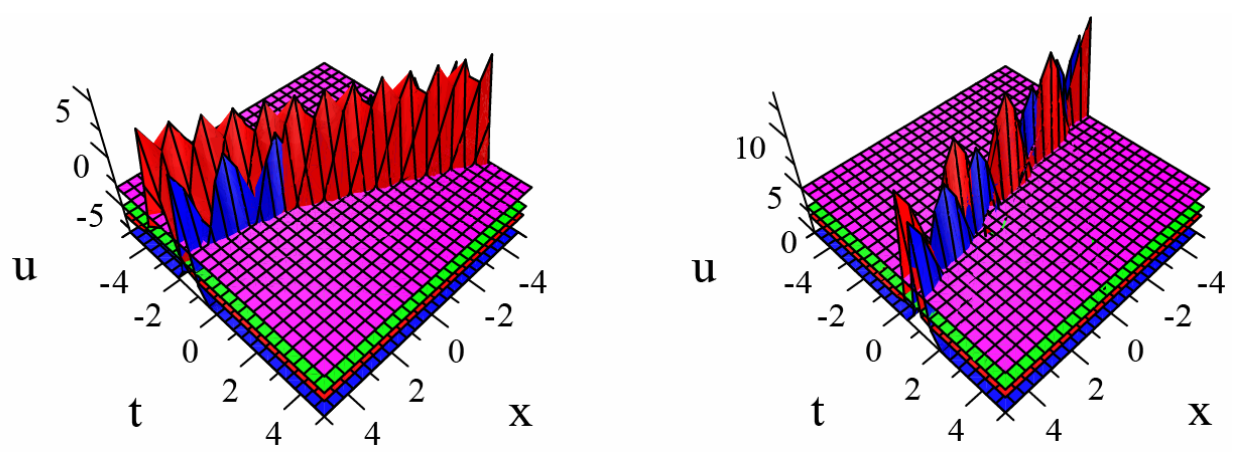

Figure 4. $u_{1}$ and $u_{2}$ in (28). 
where $c_{1} C$ is the integration constant.

Introducing (4) into (31) we have

$$
\begin{aligned}
& \left(\alpha c_{1}-\omega\right) \phi+\frac{1}{2} \alpha \phi^{2}+\beta k \phi^{\prime}+\gamma k^{2} \phi^{\prime \prime} \\
& +c_{1}\left(\frac{1}{2} \alpha c_{1}+C-\omega\right)=0 .
\end{aligned}
$$

Using the conditions (5), (6) and (14), we get (15), then we get the following two cases according to the values of $C_{1}$.

Case (1). $c_{1}=0$ :

In this case, we get the exact solutions of the tanhfunction method in the forms [13]

$$
\begin{aligned}
& u_{1}=-\frac{3 \beta^{2}}{25 \alpha \gamma}\left[1-\tanh \left(\frac{\beta}{10 \gamma}\left(x+\frac{6 \beta^{2}}{25 \gamma} t\right)\right)\right]^{2}, \\
& u_{2}=\frac{12 \beta^{2}}{25 \alpha \gamma}-\frac{3 \beta^{2}}{25 \alpha \gamma}\left[1-\tanh \left(\frac{\beta}{10 \gamma}\left(x-\frac{6 \beta^{2}}{25 \gamma} t\right)\right)\right]^{2},
\end{aligned}
$$

these solutions represent 2-dimensional surfaces in the Monge form as shown in Figure 5 for $\alpha=\beta=\gamma=1$.

Case (2). $c_{1}=\frac{2}{\alpha}(\omega-C)$ :

In this case, we have the exact solutions in the forms

$$
\begin{aligned}
u_{1}= & \frac{2 C}{\alpha}-\frac{3 \beta^{2}}{25 \alpha \gamma}\left[1-\tanh \left(\frac{\beta}{10 \gamma}\left(x-\frac{-6 \beta^{2}+50 \gamma C}{25 \gamma} t\right)\right)\right]^{2}, \\
u_{2}= & \frac{2 C}{\alpha}+\frac{12 \beta^{2}}{25 \alpha \gamma} \\
& -\frac{3 \beta^{2}}{25 \alpha \gamma}\left[1-\tanh \left(\frac{\beta}{10 \gamma}\left(x-\frac{6 \beta^{2}+50 \gamma C}{25 \gamma} t\right)\right)\right]^{2} .
\end{aligned}
$$

These relations represent surfaces whose Gaussian curvature $K$ and mean curvature $H$ are given by (35) where $a_{1}, a_{2}, k$ and $\omega$ are given in (34), thus the solutions (34) represent a family of parabolic surfaces $(K=0, H \neq 0)$, and a family of planes $(K=H=0)$ at $x=\omega t+\frac{1}{2 k} \sinh ^{-1}(-3 / 4)$ as shown in Figure 6 for
$C=0,1, \alpha=\gamma=1$; when $C=0$, we get the solutions (33).

\section{Fisher Equation}

Consider the Fisher equation

$$
u_{t}-u_{x x}-u(1-u)=0
$$

Using (2) to change (36) into the nonlinear ODE

$$
-\omega k U^{\prime}-k^{2} U^{\prime \prime}-u(1-u)=0 .
$$

Introducing (4) into (37), we get

$$
-\omega k \phi^{\prime}-k^{2} \phi^{\prime \prime}+\left(2 c_{1}-1\right) \phi+\phi^{2}+c_{1}\left(c_{1}-1\right)=0 .
$$

Using conditions (5), (6) and that $\phi_{ \pm}$satisfies the algebraic equation

$$
\left(2 c_{1}-1\right) \phi_{ \pm}+\phi_{ \pm}^{2}=0
$$

then the constant term in (38) equals to zero

$$
c_{1}\left(c_{1}-1\right)=0 \text {. }
$$

Then we have the following two cases according to the values of $c_{1}$.

Case (1). $c_{1}=0$ :

In this case, we get the exact solutions of the tanhfunction method in the form [14]

$$
u_{1,2}=\frac{1}{4}\left[1 \pm \tanh \left(\frac{1}{2 \sqrt{6}}\left(x \pm \frac{5}{\sqrt{6}} t\right)\right)\right]^{2},
$$

these solutions are plotted as shown in Figure 7 for $\alpha=\beta=1$.

Case (2). $\quad c_{1}=1$ :

In this case, we get the same exact solutions (41).

\section{Boussinesq Equation}

Consider the Boussinesq equation in the form

$$
u_{t t}-u_{x x}+\alpha\left(u^{2}\right)_{x x}+\beta u_{x x x x}=0 .
$$

Using (2), for $k=1$, to change (42) into the nonlinear ODE, we get

$$
\begin{aligned}
& K_{1,2}=0, \\
& H_{1,2}=\frac{-k^{2}\left(1+\omega^{2}\right) \operatorname{sech}^{4}(k(x-\omega t))\left(-4 a_{2}+2 a_{2} \cosh (2 k(x-\omega t))+a_{1} \sinh (k(x-\omega t))\right)}{2\left(1+k^{2}\left(1+\omega^{2}\right) \operatorname{sech}^{6}(k(x-\omega t))\left(a_{1} \cosh (k(x-\omega t))+2 a_{2} \sinh (k(x-\omega t))\right)^{2}\right)^{\frac{3}{2}},} \\
& K_{1,2}=H_{1,2}=0 \mathrm{at} x=\omega t+\frac{1}{2 k} \sinh ^{-1}(-3 / 4),
\end{aligned}
$$



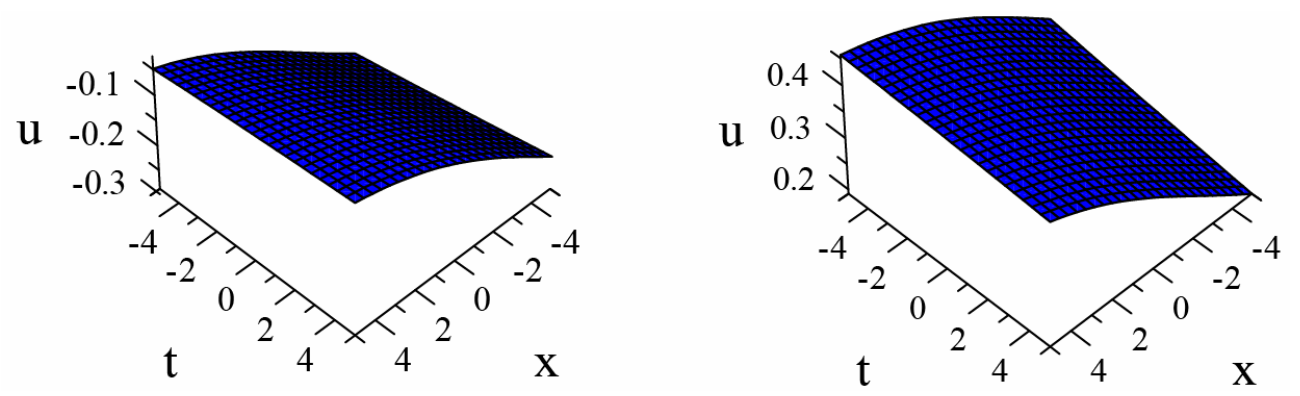

Figure 5. $u_{1}$ and $u_{2}$ in (33).
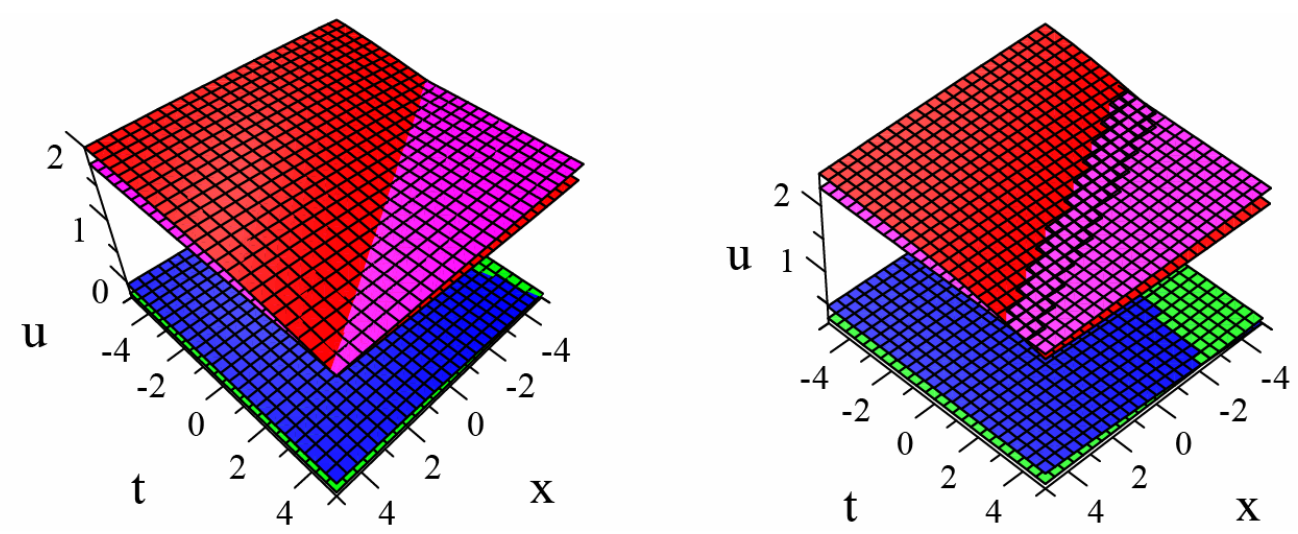

Figure 6. $u_{1}$ and $u_{2}$ in (34).
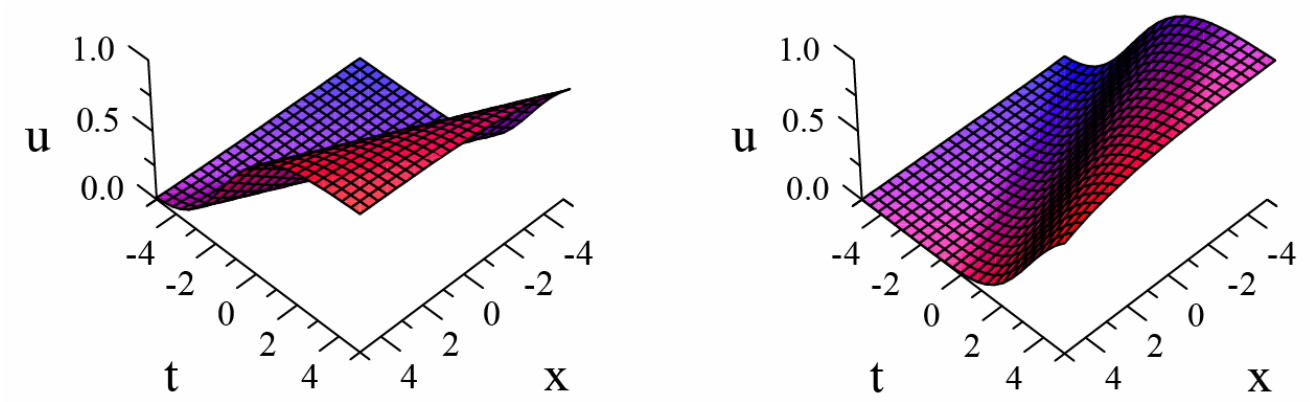

Figure 7. $u_{1}$ and $u_{2}$ in (41).

$$
\left(\omega^{2}-1\right) U^{\prime}+\alpha\left(U^{2}\right)^{\prime \prime}+\beta U^{\prime \prime \prime \prime}=0 .
$$

Integrating twice and leave the integration constant of the last integration, we have

$$
\left(\omega^{2}-1\right) U+\alpha U^{2}+\beta U^{\prime \prime}+c_{1} C=0 .
$$

Introducing (4) into (44), we obtain

$$
\left(2 \alpha c_{1}+\omega^{2}-1\right) \phi+\alpha \phi^{2}+\beta \phi^{\prime \prime}+c_{1}\left(\alpha c_{1}+C+\omega^{2}-1\right)=0 \text {. }
$$

Using the conditions (5), (6) and that $\phi_{ \pm}$satisfies the algebraic equation

$$
\left(2 \alpha c_{1}+\omega^{2}-1\right) \phi_{ \pm}+\alpha \phi_{ \pm}^{2}=0
$$

then the constant term in (45) equals to zero

$$
c_{1}\left(\alpha c_{1}+C+\omega^{2}-1\right)=0 .
$$

Then we have the following two cases according to the values of $c_{1}$.

Case (1). $c_{1}=0$ :

In this case, we get the exact solutions of the tanhfunction method in the forms [15]

$$
\begin{aligned}
& u_{1,2}=\frac{2 \beta}{\alpha}-\frac{6 \beta}{\alpha} \tanh ^{2}[x \pm \sqrt{1+4 \beta} t] \\
& u_{3,4}=\frac{6 \beta}{\alpha}-\frac{6 \beta}{\alpha} \tanh ^{2}[x \pm \sqrt{1-4 \beta} t]
\end{aligned}
$$

these solutions represent 2-dimensional surfaces in the 
Monge form as shown in Figure 8 for $\alpha=1, \beta= \pm 1$.

Case (2). $\quad c_{1}=\frac{1}{\alpha}\left(1-\omega^{2}-C\right)$ :

In this case, we get the exact solutions in the forms

$$
\begin{aligned}
& u_{1,2}=\frac{C+2 \beta}{\alpha}-\frac{6 \beta}{\alpha} \tanh ^{2}(x \pm \sqrt{1+4 \beta-2 C} t), \\
& u_{3,4}=\frac{C+6 \beta}{\alpha}-\frac{6 \beta}{\alpha} \tanh ^{2}(x \pm \sqrt{1-4 \beta-2 C} t) .
\end{aligned}
$$

These relations represent surfaces whose Gaussian curvature $K$ and mean curvature $H$ are given by (50)(51). then the solutions (49) represent a family of parabolic surfaces $(K=0, H \neq 0)$, and a family of planes

$(K=H=0)$ at

$x=\mp \sqrt{1-4 \beta-2 C} t+\frac{1}{2} \cosh ^{-1}(2)$,

$x=\mp \sqrt{1+4 \beta-2 C} t+\frac{1}{2} \cosh ^{-1}(2)$ as shown in Figure 9 for $C=0,1$ and $\alpha=1, \beta= \pm 1$; and when $C=0$, we get the solutions (48).

\section{Klein-Gordon Equation}

Consider the Klein-Gordon equation in the form

$$
u_{t t}-\alpha u_{x x}+\beta u+\gamma u^{3}=0 .
$$

Using(2) to change (52) into the nonlinear ODE

$$
k^{2}\left(\omega^{2}-\alpha\right) U^{\prime \prime}+\beta U+\gamma U^{3}=0 .
$$

Introducing (4) into (53)

$$
\begin{aligned}
& k^{2}\left(\omega^{2}-\alpha\right) \phi^{\prime \prime}+\left(\beta+3 \gamma c_{1}^{2}\right) \phi+3 \gamma c_{1} \phi^{2} \\
& +\gamma \phi^{3}+c_{1}\left(\beta+\gamma c_{1}^{2}\right)=0 .
\end{aligned}
$$

Using the conditions (5), (6) and that $\phi_{ \pm}$satisfies the algebraic equation

$$
\left(\beta+3 \gamma c_{1}^{2}\right) \phi_{ \pm}+3 \gamma c_{1}^{2} \phi_{ \pm}+\gamma \phi_{ \pm}^{3}=0,
$$

then the constant term in (54) equals to zero

$$
c_{1}\left(\beta+\gamma c_{1}^{2}\right)=0 .
$$

Then we have the following two cases according to the values of $c_{1}$.

Case (1). $c_{1}=0$ :

In this case, we obtain the solutions of the tanhfunction method in the form [7]

$$
\begin{aligned}
u_{1,2}= & \pm \sqrt{-\frac{\beta}{\gamma}} \tanh \left(\sqrt{\frac{\beta}{2\left(\omega^{2}-\alpha\right)}}(x-\omega t)\right), \\
& \frac{\beta}{2\left(\omega^{2}-\alpha\right)}>0 .
\end{aligned}
$$

these solutions are plotted as shown in Figure $\mathbf{1 0}$ for $\alpha=\beta=-\gamma=1$ and $\omega=2$.

Case (2). $c_{1}= \pm \sqrt{-\frac{\beta}{\gamma}}$ :

In this case, we get the same exact solutions (57).

\section{Conclusions}

In this paper, we introduced a new technique, by adding an integration constant and a new transformation (4) then using the tanh-function method, to obtain exact solitary wave solutions in case of the nonlinear evolution and wave equations that turn into nonlinear integrable ODEs using the wave transformation (2).

By this technique, we obtained exact solutions of the Burgers equation in (22), the KdV equation in (28), the

$$
\begin{aligned}
& K_{1,2}=0, \\
& H_{1,2}=\frac{12 \alpha^{2} \beta(2 \beta+1-C)\left(-2+\cosh (2(x \pm \sqrt{1+4 \beta-2 C} t)) \operatorname{sech}^{4}(2(x \pm \sqrt{1+4 \beta-2 C} t))\right.}{\left(\alpha^{2}+1152 \beta^{2}(1+2 \beta-C) \operatorname{sech}^{4}(2(x \mp \sqrt{1+4 \beta-2 C} t)) \tanh ^{2}(2(x \mp \sqrt{1+4 \beta-2 C} t))\right)^{\frac{3}{2}}}, \\
& K_{1,2}=H_{1,2}=0 \text { at } x=\mp \sqrt{1-4 \beta-2 C} t+\frac{1}{2} \cosh ^{-1}(2), \\
& K_{3,4}=0, \\
& H_{3,4}=\frac{-12 \alpha^{2} \beta(2 \beta-1+C)(-2+\cosh (2(x \pm \sqrt{1-4 \beta-2 C} t))) \operatorname{sech}^{4}(2(x \pm \sqrt{1-4 \beta-2 C} t))}{\left(\alpha^{2}+1152 \beta^{2}(1-2 \beta+C) \operatorname{sech}^{4}(2(x \pm \sqrt{1-4 \beta-2 C} t)) \tanh ^{2}(2(x \pm \sqrt{1-4 \beta-2 C} t))\right)^{\frac{3}{2}}}, \\
& K_{3,4}=H_{3,4}=0 \text { at } x=\mp \sqrt{1+4 \beta-2 C} t+\frac{1}{2} \cosh ^{-1}(2),
\end{aligned}
$$



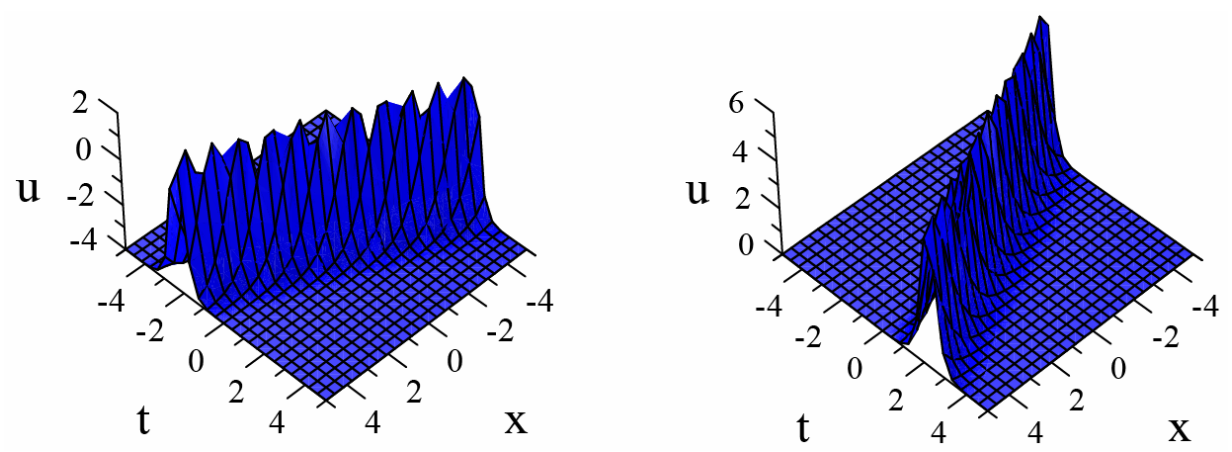

Figure 8. $u_{1}$ and $u_{2}$ in (48).
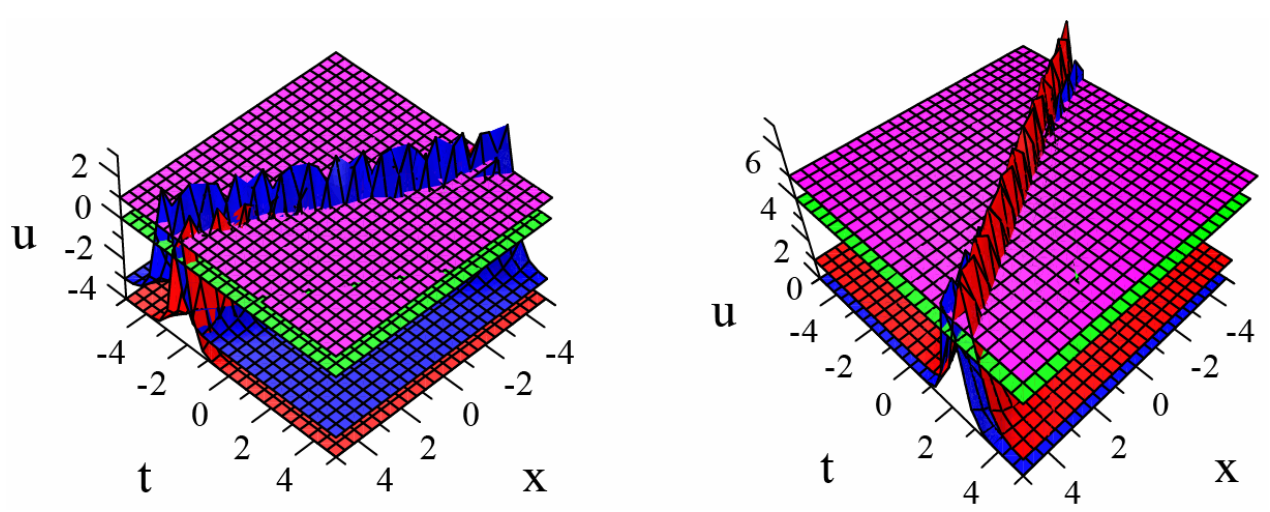

Figure 9. $u_{1}$ and $u_{2}$ in (49).
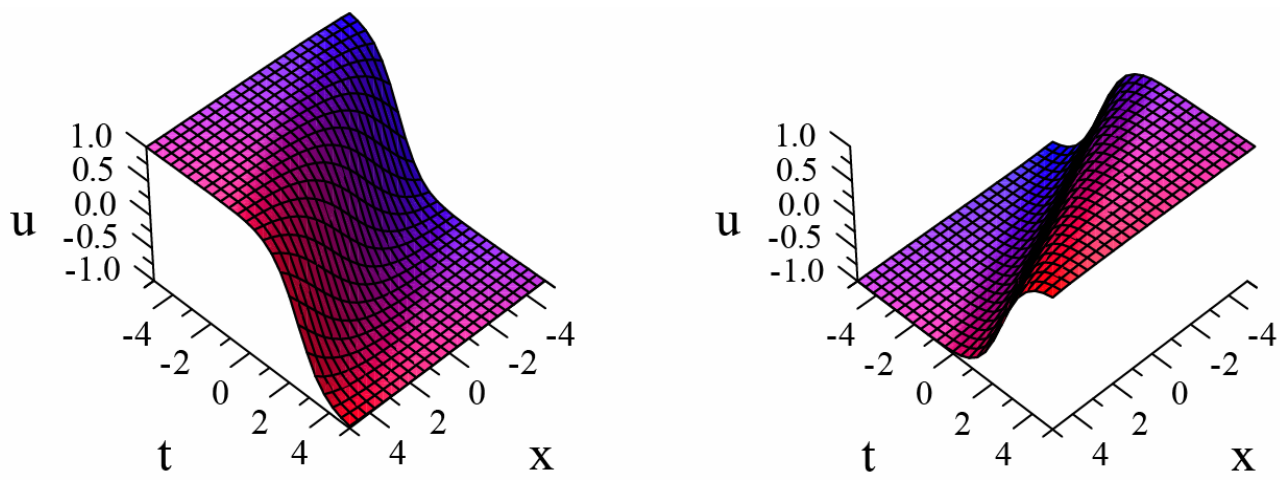

Figure 10. $u_{1}$ and $u_{2}$ in (57).

$\mathrm{KdV}$-Burgers equation in (34) and the Boussinesq equation in (49) which all give the exact solutions obtained before by the tanh-function method as a special cases [13-15]. Moreover, we discussed the geometric interpretations of the resulting exact solutions.

Also, we get the same exact solutions by using (4) then using the tanh-function method. In case of the nonlinear evolution and wave equations that turn into nonlinear non integrable ODEs using (2), Fisher and Klein-Gordon equations are considered to illustrate our technique.

The presented technique can be applied to obtain exact solutions for many nonlinear evolution and wave equa- tions.

\section{References}

[1] M. J. Ablowitz and H. Segur, "Solitons, Nonlinear Evolution Equations and Inverse Scattering," Cambridge University Press, Cambridge, 1991.

[2] R. Hirota, "The Direct Method in Soliton Theory," Cambridge University Press, Cambridge, 2004.

[3] R. Conte, "Painlevé Property," Springer, Berlin, 1999.

[4] W. Hereman, P. P. Banerjee, A. Korpel, G. Assanto, A. Van Immerzeele and A. Meerpoel, "Exact Solitary Wave Solutions of Non-Linear Evolution and Wave Equations 
Using a Direct Algebraic Method,” Journal of Physics A: Mathematical and General, Vol. 19, No. 5, 1986, pp. 607-628. doi:10.1088/0305-4470/19/5/016

[5] W. Malfliet, "Solitary Wave Solutions of Nonlinear Wave Equations,” American Journal of Physics, Vol. 60, No. 7, 1992, pp. 650-654. doi:10.1119/1.17120

[6] W. Malfliet and W. Hereman, "The Tanh Method: I Exact Solutions of Nonlinear Evolution and Wave Equations,” Physica Scripta, Vol. 54, No. 6, 1996, pp. 563-568. doi:10.1088/0031-8949/54/6/003

[7] S. A. El-Wakil, S. K. El-labany, M. A. Zahran and R. Sabry, "Modified Extended Tanh Function Method for Solving Nonlinear Partial Differential Equations," Physics Letters A, Vol. 299, No. 2-3, 2002, pp. 179-188. doi:10.1016/S0375-9601(02)00669-2

[8] E. Fan, "Extended Tanh-Function Method and Its Applications to Nonlinear Equations," Physics Letters A, Vol. 277, No. 4-5, 2000, pp. 212-218. doi:10.1080/08035250152509726

[9] Y.-T. Gao and B. Tian, "Generalized Tanh Method with Symbolic Computation and Generalized Shallow Water Wave Equation," Computers \& Mathematics with Applications, Vol. 33, No. 4, 1997, pp. 115-118. doi:10.1016/S0898-1221(97)00011-4

[10] C. Yan, "A Simple Transformation for Nonlinear Waves,” Physics Letters A, Vol. 224, No. 1-2, 1996, pp.
77-84. doi:10.1016/S0375-9601(96)00770-0

[11] Z.-Y., Yan and H.-Q. Zhang, "Auto-Darboux Transformation and Exact Solutions of the Brusselator Reaction Diffusion Model," Applied Mathematics and Mechanics, Vol. 22, No. 5, 2000, pp. 541-546. doi:10.1023/A:1016359331072

[12] W. Hereman, A. Korpel and P. P. Banerjee, "A General Physical Approach to Solitary Wave Construction from Linear Solutions,” Wave Motion, Vol. 7, No. 3, 1985, pp. 283-289. doi:10.1016/0165-2125(85)90014-9

[13] A. A. Soliman, "The Modified Extended Tanh-Function Method for Solving Burgers-Type Equations,” Physica A: Statistical Mechanics and Its Applications, Vol. 361, No. 2, 2006, pp. 394-404 doi:10.1016/j.physa.2005.07.008

[14] A.-M. Wazwaz, "The Extended Tanh Method for Abundant Solitary Wave Solutions of Nonlinear Wave Equations," Applied Mathematics and Computation, Vol. 187, No. 2, 2007, pp. 1131-1142. doi:10.1016/j.amc.2006.09.013

[15] A.-M. Wazwaz, "New Travelling Wave Solutions to the Boussinesq and the Klein-Gordon Equations," Communications in Nonlinear Science and Numerical Simulation, Vol. 13, No. 5, 2008, pp. 889-901. doi:10.1016/j.cnsns.2006.08.005 